\title{
Cycle Frequency Estimation of Time/Frequency Diversity Transmissions in a Dense Noise Environment
}

\author{
JoEllen Wilbur \\ Signal and Image Processing Branch, Code 2230 \\ Naval Coastal Systems Center \\ Panama City Beach, FL 32407-5000, USA
}

\begin{abstract}
A cycle rate estimation technique for weak diversity transmissions in noise is presented. The processing procedure is based on performing a weighted-average DWD inner product operation at the input to a discrete Fourier analyzer followed by a low frequency block then a weighted running sum across the FFT analyzer output. Fourier transformation across overlapping blocks of the summed outputs are then averaged to produce a cycle frequency estimate. The cycle frequency estimator is tested on a frequency hopped Welch 11 code Costas array in OdB SNR and on a dual-tone frequency hop/4-ary frequency shift keyed signal where random time jitter and Gaussian pulse shaping have been employed.
\end{abstract}

\section{INTRODUCTION}

A common denominator to modern pulse modulated spread spectrum and diversity signals is the application of some form of constant rate modulation. By definition, a signal of this form is cyclostationary and subject to cycle tone identification. Spectral redundancy methods employed for cycle tone identification of spread spectrum and diversity transmissions have recently received considerable attention in the literature[ 1 and references therein].

Fourier transformation of the WD estimator has been shown to produce cyclic correlations that correspond to the modulation rate of the spreading function of spread spectrum and diversity signals used in underwater data telemetry[2].

In this paper a cycle frequency estimator for frequency key pulse modulated and diversity signals is presented. The estimator is derived through Fourier transformation over time of a bilinear pseudo-Wigner distribution time-evolutionary spectrum (TES) estimator to yield a conjoint frequency-frequency correlation, or cycle spectrum, signal representation. Fourier transformation over temporal window centers of the pseudo-Wigner distribution maps stationary noise to the frequency correlation axis, nonstationary noise is distributed, and the frequency keyed modulation on the signal produces cyclic correlations called cycle frequencies that dominate when the cycle spectrum is integrated over frequency.

\section{WIGNER ANALYSIS OF CYCLIC SPECTRAL SPREADING}

The Wigner distribution (WD) is a bilinear $t$ to $t-\omega$ transformation of the form [3]

$$
W_{x x}(t, \omega)=F_{\tau \rightarrow \omega}\left\{x\left(t+\frac{\tau}{2}\right) x\left(t-\frac{\tau}{2}\right)\right\}
$$

where $x(t)$ is a time history and $F_{\tau \rightarrow \omega}($. $\}$ denotes the Fourier transform over $\tau$. The WD defines a timefrequency estimator that provides a representation of signal concentration over the $t-\omega$ plane. The WD is in fact one member of a large class of timeevolutionary spectral estimates (TES), i.e. the Cohen class [3], that serve to represent signal concentration simultaneously over time and frequency. Fourier transformation of the signal TES over time yields an estimate of the correlated spectral spreading in the signal. For a cyclostationary signal Fourier transformation will produce cyclic correlations in the spectral spreading of the signal.

In practical applications the pseudo-WD is computed in which an ensemble of WD functions are defined by moving a window of length $T$ over the signal and the pWD is defined via observation along the window centers to yield[3]

$\tilde{W}_{x x}(t, \omega)=\int_{-\infty}^{\infty} x\left(t+\frac{\tau}{2}\right) x^{*}\left(t-\frac{\tau}{2}\right) p\left(\frac{\tau}{2}\right) p\left(-\frac{\tau}{2}\right) e^{-j \omega \tau} d \tau$

where $p(t)$ is a window of length T. The pWD can be seen from (2) to define the TES in (1) where $w(\tau, \sigma)=p\left(\frac{\tau}{2}\right) p\left(-\frac{\tau}{2}\right)$.

U.S. Government work not protected by U.S. copyright. 
The Wigner distribution has been shown to form a sound basis for representing nonstationary acoustic returns over the t- $\omega$ plane. Naturally frequency key pulsed signals fall into this category and are cyclostationary. Fourier transformation over window centers in the pWD defined in (2) can be shown to equate to the time-variant cyclic spectrum evaluated along the center of a moving average estimate[4]. More specifically, the cyclic periodic autocorrelation [5] for a cyclostationary signal is defined via an exponentially weighted moving average in time on the signal time-domain inner product. The time-variant cyclic spectrum for cyclostationary processes is defined as the Fourier transform over product lags of the cyclic periodic autocorrelation[5], i.e.

$S_{x x}^{\sigma}(t, \omega) \doteq F_{\tau \rightarrow \omega}\left\{\frac{1}{T} \int_{t-\frac{T-|\tau|}{T}}^{t-\frac{T+|\tau|}{T}} x\left(u+\frac{\tau}{2}\right) X^{*}\left(u-\frac{\tau}{2}\right) e^{-j \sigma u} d u\right\}$.

Evaluation of the time variant cyclic spectrum along the center of the moving average estimate can be shown to reduce to [4]

$$
S_{x x}^{\sigma}=\int_{-\infty}^{\infty} W_{h h}(0, \omega-\xi) \int_{-\infty}^{\infty} W_{x x}(t, \xi) e^{-j \sigma t} d \omega d \xi
$$

for $h(t)$ a rectangular pulse of length $\mathrm{T}$ and $W_{x x}(t, \omega)$ and $W_{h k}(t, \omega)$ respective WD functions defined in (1). Equation (4) equates to the following relation

$$
S_{x x}^{\sigma}(\omega)=\int_{-\infty}^{\infty} e^{-j \sigma t} \tilde{W}_{x x}(t, \omega) d t
$$

where $\tilde{W}(t, \omega)$ is the pWD defined in (2) for a rectangular window of length $\mathrm{T}$ and $S_{x x}^{\sigma}(\omega)$ is the time-variant cyclic spectrum evaluated along $\mathrm{t}=0$. Transformation of the pWD over time maps the stationary noise, which has uncorrelated spectral spreading, to $\sigma \cong 0$. Nonstationary noise is distributed randomly. For a cyclostationary signal, spectral peaks arise as dominant lobes in the $\sigma-\omega$ plane at integer multiples of the signal cycle frequency which corresponds to the reciprocal of the modulation rate. In a diversity signal integration of the cycle spectrum over $\omega$ averages out the nonstationary noise components and reinforces cycle tones.

\section{CYCLE FREQUENCY ESTIMATOR AND EXAMPLES}

M-ary frequency shift keying (MFSK) defines pulse coded signalling over $M$ frequency bins and can be designed for noncoherent reception. An MFSK diversity trApplication of the low frequency block is used to elliminate the dc cross-term contributions associated with the DWD computation. ansmission is defined as below,

$$
\begin{gathered}
x_{M F S K}(t)=\sum_{m=1}^{L} \cos \left(\omega_{c} t+a_{m} \omega_{m} t\right) g\left(t-m T_{c}\right) \\
g(t)=\sum_{n=0}^{N} p(t) \cos \left(\omega_{n} t\right)
\end{gathered}
$$

where $T_{c}$ is the time between frequency keys, $N$ is the number of frequencies in each keyed subpulse, $p(t)$ is a pulse of length $T_{p}<T_{c}, \omega_{m}$ is the minimum frequency displacement, and the $a_{k}$ 's assume the values $\pm 1, \pm 3, \ldots, \pm(M-1)$ via a pseudo-random selection. Signal diversity provides tolerance to channel fading and multipathing given proper spacing between the energy loci of the frequency keyed subpulses in the $t-\omega$ plane[2].

The discrete-WD (DWD) of a frequency keyed pulse transmission designed using a Welch 11 code Costas array is given in Figure 1. The most general form of the DWD expresses as[6,7]

$$
W_{x x}(n, k)=\sum_{m=-\frac{N}{2}+1}^{\frac{N}{2}-1} p|m|^{2} i_{x x}(n, m) e^{-j \frac{2 \pi k m}{N}}-R_{x x}(0)
$$

$$
\begin{gathered}
i_{x x}(n, m)=\frac{1}{2 L-1} \sum_{l=-L+1}^{L-1} w(l) x(n+l+m) x^{*}(n+l-m) \\
R_{x x}(0)=\frac{1}{2 L-1} \sum_{l=-L+1}^{L-1} g(l)|x(n+l)|^{2}
\end{gathered}
$$

where $L=1$ reduces to the unsmoothed DWD corresponding to (2). The smoothing operation on the DWD inner product defined in ( 7 ) provides noise immunity and cross-term reduction.

The cycle spectrum is obtained via Fourier transformation over time slices, or window centers, of the DWD. The cycle frequency estimate is computed as below,

$$
\tilde{S}_{x x}(\sigma)=\sum_{i=1}^{l} \xi_{x x}^{i}(\sigma)
$$




$$
S_{x x}^{i}(\sigma)=\sum_{n=0}^{N^{\prime}-1} e^{-j \frac{2 \pi 0 n}{N^{\prime}}} \sum_{k=K_{d k}}^{\frac{N}{2}-1} W_{x x}(n, k)
$$

for N' the number of window center positions, I the number of segmented blocks in the signal and $K_{d c}$ the upper cutoff of a low frequency block. Application of the low frequency block is used to elliminate the dc cross-term contributions associated with the DWD computation.

Notice, for computational simplicity, transformation of the DWD across window centers has been interchanged with the summing operation over frequency, reducing the number of FFT operations from $N+N '+1$ to $N+2$. Figure 2 illustrates the cycle frequency estimate of the Welch 11 code Costas array illustrated in Figure 1 where the data has been segmented into two consecutive blocks. The stationary part of the signal correlation, i.e. $\sigma \equiv 0$, has been removed from the cycle frequency estimates. The regular repetition of the keying pattern produces cyclic correlations that arise as dominant lobes at integer multiples of the cycle frequency or, equivalently, the reciprocal of the keying rate. Figure 3 illustrates the cycle frequency for the case of a $\mathrm{OdB}$ signal-to-noise ratio (SNR). Figure 4 illustrates the cycle frequency for a OdB SNR dual tone frequency hop/4-ary frequency shift key (FH/4FSK) where the start time between frequency keys has been randomly jittered by $10 \%$ within a given bin and Gaussian pulse shaping has been applied to the hopped subpulses.

Given the Fourier transform relation in mapping from the DWD to the cycle spectrum it becomes clear that excessive temporal smoothing corresponds to filtering of the cycle frequencies. More specifically, given $D F T_{a \rightarrow b}^{P}\{$.$\} denotes a \mathrm{P}$ point discrete Fourier transform from a to b, Equation (8) can be rewritten as

$$
\begin{gathered}
S_{x x}(\sigma)=G_{2 L}(-\sigma) \sum_{k=K_{d k}}^{\frac{N}{2}-1} S_{x x}^{k}(\sigma) \\
G_{2 L}(-\sigma)=\frac{1}{2 L-1} \sum_{l=-L+1}^{L-1} g(l) e^{j \frac{2 \pi \sigma l}{N^{*}}} \\
S_{x x}^{k}(\sigma)=D F T_{m \rightarrow k}^{N}\left\{D F T_{n \rightarrow \sigma}^{N^{*}}\left\{x(n+m) x^{*}(n-m)\right\}\right\}
\end{gathered}
$$

where $S_{x x}^{k}(\sigma)$ is the cycle spectrum estimate obtained via Fourier transformation over the unsmoothedDWD. Figures 5 and 6 give respective cycle frequency plots for the case $\mathrm{L}=\mathrm{N} / 2$.

\section{CONCLUDING REMARKS}

An efficient processing procedure to extract cycle rate information from weak diversity signals in a high noise environment has been described. The cycle rate estimator is able to identify cycle tones in weak diversity telecommunications transmissions and frequency hopped Costas array signals in OdB SNR even after $10 \%$ random time jitter and Gaussian pulse shaping have been imposed on the signals. Multitone transmissions require a smaller number of time bins to be searched to achieve cycle tone identification.

\section{ACKNOWLEDGMENTS}

This work was supported under a Naval Coastal Systems Center's Independent Research project sponsored by the ONR.

\section{REFERENCES}

[1] W.A. Gardner, "Exploitation of Spectral Redundancy in Cyclostationary Signals," IEEE Sig. Proc. Magazine, vol. 8, no. 2, pp. 14-36 April 1991.

[2] J. Wilbur and J. Bono, "Wigner/Cycle Spectrum Analysis of Spread Spectrum and Diversity Transmissions," IEEE J. Ocean Engineering 16, 98-106 (1990).

[3] T.A.C.M. Claasen and W.F.G. Mecklenbrauker, "The Wigner Distribution--A Tool for TimeFrequency Signal Analysis, Parts I and III," Philips, J. Res., vol. 35, pp. 217-389, 1980.

[4] J. Wilbur and R.J. MDonald, "Nonlinear Analysis of Cyclically Correlated Spectral Spreading in Modulated Signals," submitted to J. Acoust. Soc. Am.

[5] W. A. Gardner, Statistical Spectral Analysis: A Nonprobabalistic Theory, Prentice Hall, Inc., New Jersey, 1988.

[6] L. Jacobson and H. Wechsler, "The Composite Pseudo Wigner Distribution (CPWD): a Computable and Versatile Approximation to the Wigner Distribution (WD)," Proc. Int. Conf. Acoust., Speech, Sig. Processing, vol. 1, pp. 254-256, 1983.

[7] P. Flandrin, W. Martin, and M. Zakharia, "On a Hardware Implementation of the Wigner-Vill Transform," in Dig. Sig. Processing-84, V. Cappelini and A.G. Constantinedes, Eds. Elsevier, 1984. 


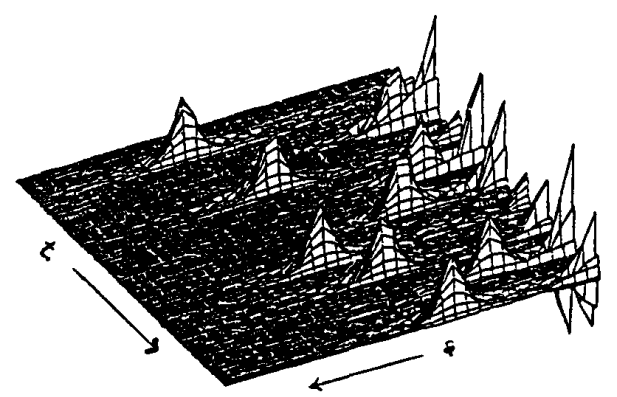

Figure 1. DWD of 1040 point frequency hop generated using a Welch 11 code Costas array $(\mathrm{N}=64$, $\mathrm{L}=2$ ).

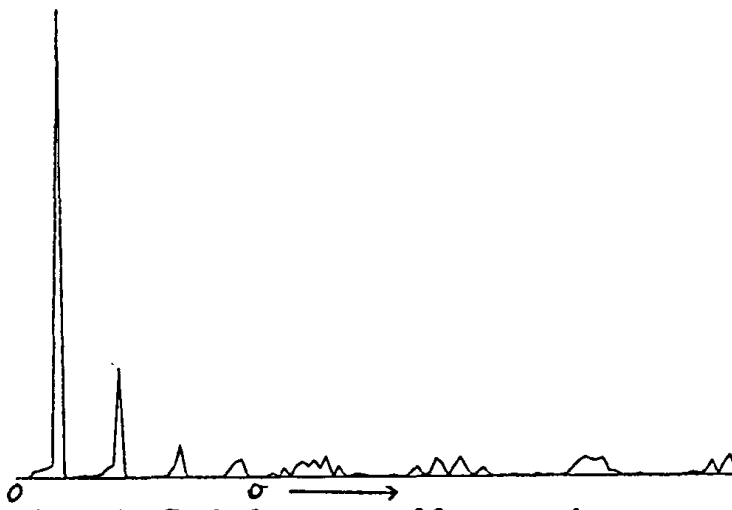

Figure 2. Cycle frequency of frequency hop generated using Welch 11 code Costas array $(\mathrm{N}=64, \mathrm{~L}=2$, $\mathrm{I}=2$ ). [nonstationary part]

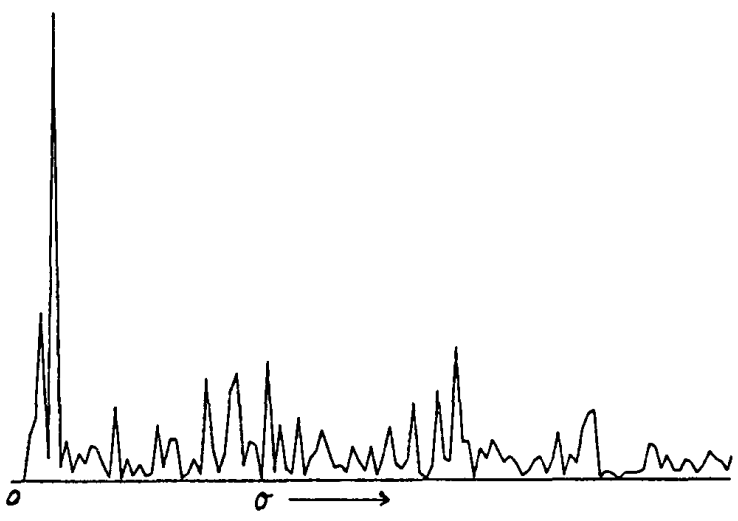

Figure 3. Cycle frequency of frequency hop generated using Welch 11 code Costas array (SNR $=0 \mathrm{~dB}$, $\mathrm{N}=64, \mathrm{~L}=2, \mathrm{I}=2$ ).[nonstationary part]

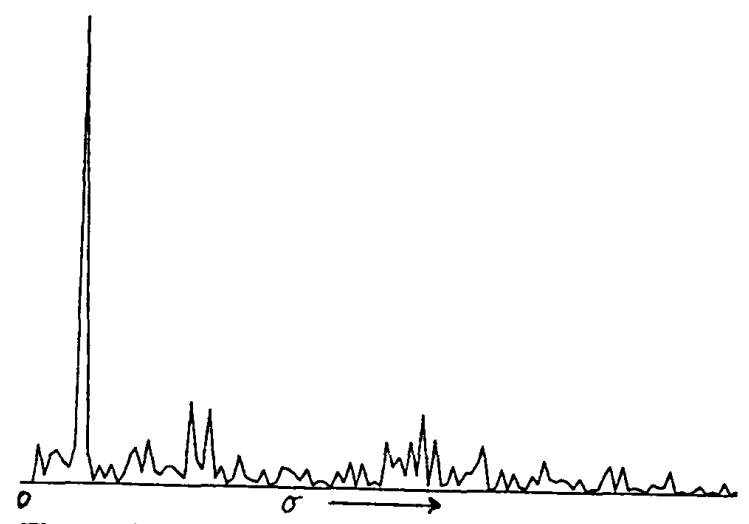

Figure 4. Cycle frequency estimate of dual tone FH/4FSK with $10 \%$ random time jitter and Gaussian pulse shaping on the signal subpulses $(S N R=0 \mathrm{~dB}$, $\mathrm{N}=64, \mathrm{~L}=2, \mathrm{I}=2$ ). [nonstationary part]

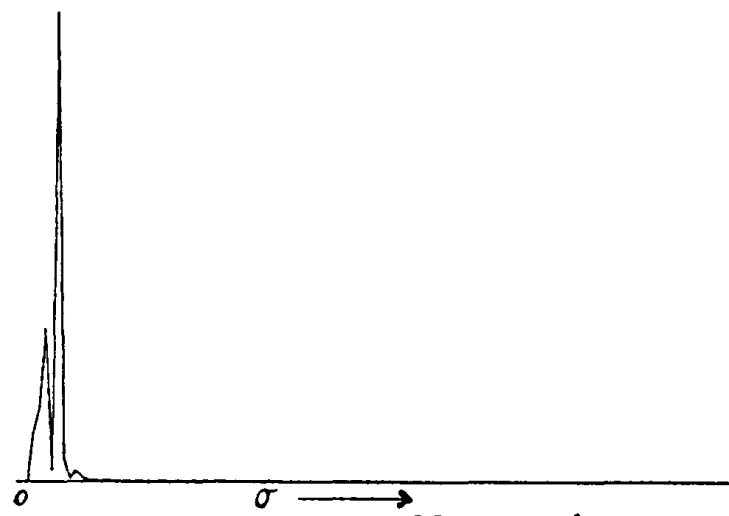

Figure 5. Cycle frequency of frequency hop generated using Welch 11 code Costas array (SNR $=0 \mathrm{~dB}$, $\mathrm{N}=64, \mathrm{~L}=32, \mathrm{I}=2$ ). [nonstationary part]

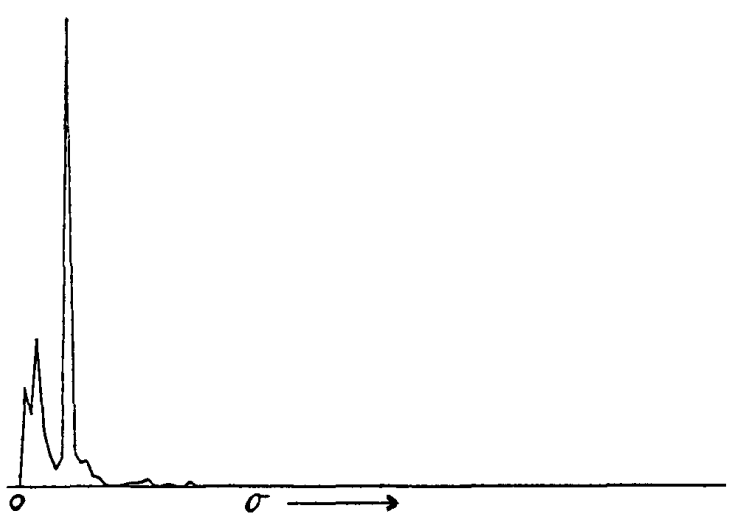

Figure 6. Cycle frequency estimate of dual tone FH/4FSK with $10 \%$ random time jitter and Gaussian pulse shaping on the signal subpulses ( $\mathrm{SNR}=0 \mathrm{~dB}$, $\mathrm{N}=64, \mathrm{~L}=32, \mathrm{I}=2$ ). [nonstationary part] 